\title{
Old LMC Globular Clusters: Reticulum
}

\author{
Gianni Marconi \\ ESO-Chile, Alonso de Cordova 3107 Casilla 19001, Santiago de Chile, \\ Chile \\ Vincenzo Ripepi \\ Naples Observatory, Via Moiarello 16, 80131 Napoli, Italy \\ Gloria Andreuzzi, Giuseppe Bono, Roberto Buonanno \\ Rome Observatory, Via Frascati 33, 00040 Monte Porzio Catone, Italy \\ Santi Cassisi \\ Teramo Observatory, Via M. Maggini, 64100 Teramo, Italy
}

\begin{abstract}
We present a preliminary analysis of homogeneous, deep and accurate multiwavelength $\mathrm{U}, \mathrm{B}, \mathrm{V}$, and I data of the old LMC cluster Reticulum. The comparison between theory and observations seems to suggest that the reddening toward this cluster is a factor of 2 larger than suggested in the literature. Both cluster distance and age are in agreement with the canonical values. Finally, the main goals of this project are briefly discussed.
\end{abstract}

\section{Introduction}

Reticulum is one of the old LMC clusters and plays a key role in constraining both evolutionary and pulsational properties of low-mass stars. Compared with the other clusters in the Magellanic Clouds it has a very low central density, therefore the photometry is not affected by crowding. The main aim of our project is to supply accurate estimates of the cluster's reddening, and the mean magnitude of RR Lyrae (32) and turn-off stars. We are interested in these observables because the evaluations of mean reddening and LMC distances available in the literature are still controversial. Moreover, we still lack a firm age determination for this cluster. To accomplish this project we collected new accurate and homogeneous multiband optical $(\mathrm{U}, \mathrm{B}, \mathrm{V}, \mathrm{I})$ and NIR $(\mathrm{J}, \mathrm{K})$ data.

\section{Observations \& Calibrations}

Multicolor (UBVIJK) data have been acquired under exceptional seeing conditions $\leq 0.6$ " at the ESO NTT telescope in two different observing runs (Dec 1999 and Dec 2000). To properly sample the light curve of RR Lyrae variables, 

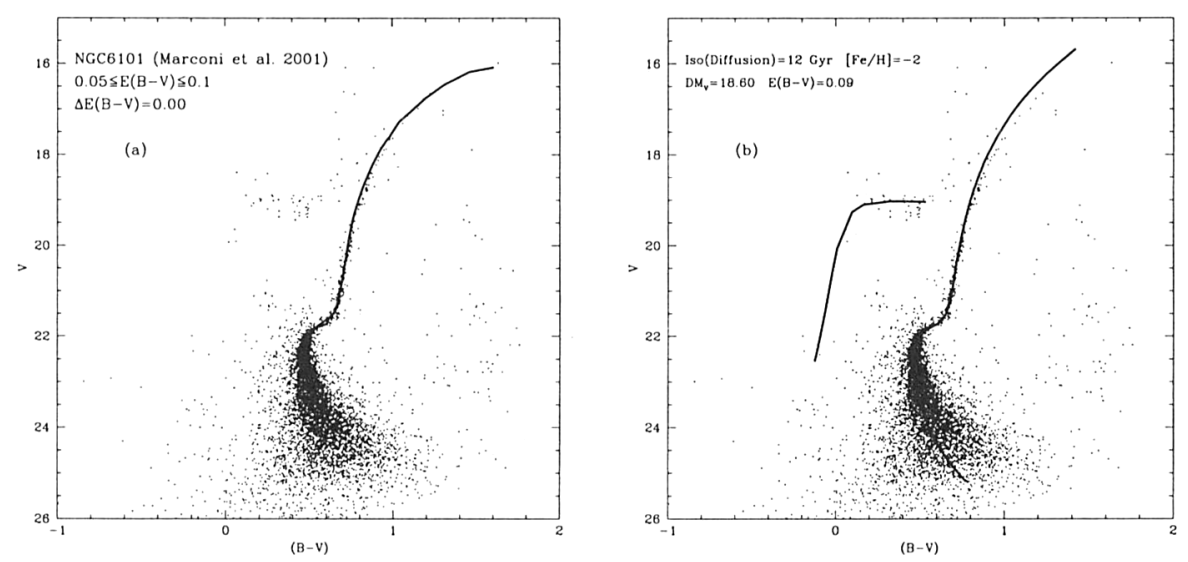

Figure 1. CMD (V,B-V) of Reticulum. The left panel shows the comparison bwteeen the cluster data and the mean fiducial line of NGC6101, while the right one the comparison with an isochrone of 12 Gyr.

we collected 20 short exposures for each optical and infrared band. Moreover, to improve the accuracy of the faint main-sequence photometry, two deep B and V exposures were secured. To obtain very accurate absolute calibrations a set of Landolt stars covering the color range of typical globular cluster stars have been observed during the same nights. In order to determine the extinction curve the standards were observed in each night and at different airmasses. Optical data were reduced by using PSF-fitting photometry (DAOPHOT-ALLSTAR).

\section{Comparison with NGC 6101 and Isochrones}

Fig. 1a shows the CMD of Reticulum derived by using deep B and V exposures. In order to derive an empirical estimate of the cluster reddening we selected the Galactic globular NGC 6101, since the two clusters present similar metallicities $([\mathrm{Fe} / \mathrm{H}] \approx-1.8 \mathrm{dex})$. The agreement between the cluster data and the mean fiducial line (Marconi et al. 2001) for NGC 6101 suggests that the two clusters should have the same reddening. Current empirical estimates suggest for NGC 6101 a mean reddening of $E(B-V)=0.06 \pm 0.02$ mag (Sarajedini et al. 1991). Note that according to the cluster reddening compilation by Dutra \& Bica (2000) the reddening estimates for this cluster range from 0.05 to 0.1 mag. This finding supports the evidence that the reddening toward Reticulum could be somewhat larger than derived by Walker (1992), i.e., $\mathrm{E}(\mathrm{B}-\mathrm{V})=0.03 \pm 0.02$.

Even though detailed V and I HST data for six old LMC clusters have been recently presented by Olsen et al. (1998) we still lack accurate photometric data to firmly estimate the age of Reticulum. The quality of our optical data allows us to perform an accurate comparison with theoretical isochrones. Fig. 1b shows the comparison of cluster data with an isochrone of $12 \mathrm{Gyr},[\mathrm{Fe} / \mathrm{H}]=-2 \mathrm{dex}$, and 
$\mathrm{Y}=0.23$ (Cassisi et al. 1998). Theoretical predictions were transformed into the observational plane by adopting the bolometric corrections and color - temperature relations by Castelli et al. (1997). The evolutionary tracks adopted to construct this isochrone account for element diffusion. By adopting a distance modulus of $18.6 \mathrm{mag}$ the cluster data are fitted only by assuming a reddening approximately equal to $0.09 \mathrm{mag}$. Within current uncertainties for cluster distance, the reddening we found roughly agrees with estimates based on empirical data. We are aware that spectroscopic measurements suggest that Reticulum is slightly more metal-rich $([\mathrm{Fe} / \mathrm{H}]=-1.71 \pm 0.09 \mathrm{dex}$, Suntzeff et al. 1992). However, even adopting more metal-rich and/or slightly older isochrones does not substantially change the reddening value. The zero-age horizontal branch for the same metallicity and constructed by adopting the same theoretical framework is also plotted in Fig. 1b. We find that by adopting the same distance and reddening the theoretical predictions fit the observed distribution of HB stars. Note that the spread in magnitude of RR Lyrae stars is fictitious, since we have not yet derived the mean magnitudes.

\section{Conclusions and future work}

Comparison between theory and observations suggests that Reticulum is of the same age as genuine old Galactic globular clusters and the reddening seems to be approximately a factor of two larger than previously estimated in the literature. In the near future, using also the $U$ band data, we plan to supply a new independent estimate of the cluster reddening by measuring the individual reddening of cluster RR Lyrae by adopting the reddening free index "Q". We are also interested in providing a new distance determination using not only optical mean magnitudes but also the $K_{s}$ mean magnitudes. We also plan to supply the cluster distance by using the K-band Period-Luminosity relation. Current theoretical and empirical evidence suggest in fact that this method should supply very accurate distance determinations for both cluster and field RR Lyrae stars. As a matter of fact, K-band magnitudes are marginally affected by reddening and present a mild dependence on metallicity. Finally let us mention that the data we collected will allow us to evaluate for the the first time the LMC distance using mean NIR RR Lyrae magnitudes.

\section{References}

Cassisi, S., Castellani, V., degl'Innocenti, S., Weiss, A., 1998, A\&AS, 129, 267

Castelli, F., Gratton, R.G., Kurucz, R.L., 1997, A\&A, 324, 432

Dutra, C.M., \& Bica, E., 2000, A\&A, 359, 347

Marconi, G., Andreuzzi, G., Pulone, L., et al., 2001, in preparation

Olsen, K.A.G., Hodge, P.W., Mateo, M., et al., 1998, MNRAS, 300, 665

Sarajedini, A., \& Da Costa, G.S., 1991, AJ, 102, 628

Suntzeff, N.B., Schommer, R.A., Olszewski, E.W., Walker, A.R., 1992, AJ, 104, 174

Walker, A.R., 1992, AJ, 103, 1166 